\title{
Para un currículum de educación en valores
}

\section{Towards a Curriculum Based on the Education of Values}

\author{
Josep María Puig Rovira ${ }^{1}$ \\ Xus Martín García²
}

\section{Resumen}

La educación en valores suele buscar fundamentos ciertos y seguros a partir de los cuales orientar, explicar y justificar la intervención de los educadores. La crítica a la modernidad cuestiona la posición de superioridad que se le ha otorgado a la teoría respecto a la práctica educativa y plantea un nuevo escenario desde el que pensar la educación. En este artículo se defiende la conveniencia de abandonar las seguridades y verdades absolutas en beneficio de una educación en valores que parta de los interrogantes de la realidad, una educación preocupada por ayudar a las personas a aprender a vivir. Para ello se propone un currículo de educación en valores que se aleje de los contenidos acabados y, en su lugar, ofrezca un conjunto de prácticas morales que permitan a los jóvenes enfrentarse a la realidad y decidir qué aspectos deben mantenerse y cuál va a ser su aportación al momento actual que vive la sociedad.

Palabras clave

Educación en valores, prácticas morales, educación para la ciudadanía, deliberación moral, virtudes cívicas.

\section{Abstract}

Education on values looks for a certain and secure foundation from which to guide, explain and justify the intervention of educators. The critique of modernity questions the superiority that has been given to the theory about educational practice, and poses a new scenario for thinking about education. The article defends the desirability of abandoning securities and absolute truths for the benefit of an education in values starting with the questions of reality, an education that is concerned about helping people to learn how to live. In order to achieve these objectives, we propose a curriculum that is away from fixed contents. Instead, we propose a curriculum that offers a set of moral practices that allow young people to face reality and decide what aspects should be kept and which one will be their contribution to the current situation of society.

\section{Keywords}

Education, moral practices, citizenship education, moral deliberation, civic virtues.

Artículo recibido el 27 de octubre de 2014 - aprobado el 13 de noviembre 2014

1 Facultad de Educación de la Universidad de Barcelona. Correo electrónico: joseppuig@ub.edu

2 Facultad de Educación de la Universidad de Barcelona. Correo electrónico: xusmartin@ub.edu 
El objetivo de este escrito es desarrollar una propuesta curricular de educación en valores. Una propuesta que, como pedía Stenhouse (1984), no se limite a proponer un conjunto de ideas teóricas, sino que tenga la deferencia hacia el profesorado de convertirlas en un currículo que pueda aplicarse y probarse en las aulas. Para avanzar hacia una propuesta de esta naturaleza nos planteamos tres interrogantes: a) ¿desde dónde pensar la educación en valores?, b) ¿qué marco de ideas puede orientar el desarrollo de un currículo de educación en valores?, y c) ¿cómo traducir esas ideas a actividades escolares? En las páginas siguientes vamos a intentar responder a cada uno de estos temas.

Sin embargo, antes de abordar cada una de estas cuestiones, conviene hacer un breve apunte conceptual. Hablamos de educación en valores para referirnos a un núcleo de cuestiones que son comunes con otros conceptos que consideramos próximos. Denominaciones como educación para la ciudadanía, educación para los derechos humanos, educación democrática, educación ética, educación moral $y$ algunas otras, no se refieren exactamente a lo mismo, pero en gran medida coinciden tanto en el fondo de las problemáticas que plantean, como en el modo que deberían tratarse didácticamente en las aulas. Por tanto, al adoptar la denominación de educación en valores entendemos que nos estamos refiriendo a un núcleo temático común a múltiples denominaciones cercanas $y$, en consecuencia, los contenidos que vamos a desarrollar pueden ser útiles para cualquiera de estos ámbitos conceptuales.

Dicho esto, volvamos a las preguntas iniciales: ¿desde dónde pensar la educación en valores?, ¿qué ideas nos ayudarán a desarrollar un currículum?, y ¿cómo concretarlo en prácticas escolares?

\section{Ni fundacionalismo ni reduccionismo}

La pregunta por el lugar desde dónde pensar la educación en valores no dista mucho de la que también se plantea el conjunto del pensamiento pedagógico: cómo producir y cómo justificar el saber sobre la educación. En ambos casos, la respuesta ha recurrido a una posición fundacionalista $\mathrm{o}$, manteniendo una estructura parecida y cambiando tan solo el contenido en el que apoyarse, se ha caído en un reduccionismo, normalmente de índole científica.

Wilfred Carr (2007) es uno de los autores que denunció con más fuerza lo insostenible de ambas posturas. En su opinión, la teoría de la educación siempre ha pretendido realizar dos tareas relacionadas. La primera, de índole filosófica, destinada a identificar los fundamentos ciertos y seguros a partir de los cuales fuese posible obtener principios pedagógicos objetivos y racionales. La segunda tarea, de naturaleza aplicada, debía guiar la práctica de los educadores reemplazando sus prejuicios y creencias subjetivas por un conocimiento objetivo generado a partir de la teoría educativa. En definitiva, Carr critica dos cuestiones: la voluntad de derivar la teoría educativa a partir de un fundamento seguro, sea de raíz metafísica o de naturaleza científica, y la pretensión de dirigir la práctica que llevan a cabo los educadores a partir de un saber teórico previamente bien fundamentado.

Tras la crítica posmoderna y el cerco a la idea de verdad (Lyotard, 1979), para Carr no resulta posible mantener la estrategia de búsqueda de un fundamento anterior y seguro desde el cual desarrollar un campo de conocimiento como el educativo. Una estrategia que, por otra parte, deja la reflexión pedagógica en una situación de gran dependencia respecto de un saber considerado superior. $\mathrm{Y}$, en relación a la segunda tarea, también niega la supuesta capacidad de la teoría educativa para orientar la práctica, y lo hace mostrando la insuperable distancia que separa la acción práctica de la teoría. Estamos de nuevo ante una consecuencia inaceptable: la posición de superioridad en la que quedan los teóricos de la educación respecto a los educadores ocupados en sus prácticas cotidianas. $\mathrm{Ni}$ el fundacionalismo ni el reduccionismo pedagógico son deseables para desarrollar el saber pedagógico y, como veremos, tampoco son estrategias adecuadas para pensar la educación en valores.

Una argumentación similar nos ofrece Hilary Putnam (2013) en su obra Ética sin ontología. El autor no considera plausible la existencia de un 
conjunto de ideas, formas o verdades que la filosofía debe encargarse de descubrir y que actúan como justificación última de las valoraciones y las obligaciones morales de individuos y sociedades. El fundacionalismo ético conecta la vida moral al conocimiento de las formas del bien que se supone existen con anterioridad e independencia a cualquier realidad mundana. La reflexión ética consiste en la búsqueda y el conocimiento de estas verdades preexistentes que deben dar forma a la vida humana. Con Putnam, consideramos que el hecho de poder dar nombre y desarrollar teóricamente ciertas ideas sobre el bien no permite creer que tales conceptos existen con anterioridad a su aparición y su evolución en el seno de las sociedades humanas. Por tanto, no parece posible pensar la educación en valores desde la búsqueda y el conocimiento de alguna idea ultramundana del bien.

Si el fundacionalismo ha sido considerado durante mucho tiempo el modo mayor de justificar la educación en valores, el reduccionismo ha heredado su lógica, aunque la seguridad venga en este caso desde el conocimiento científico. Pensar de manera reduccionista es afirmar que aquello que deseamos conocer se explica del todo gracias a un saber, normalmente científico, que tenemos a disposición, aunque este conocimiento tan solo aborde alguno de los aspectos presentes en el tema que nos preocupa. En nuestro caso, estamos ante una posición reduccionista cuando explicamos la educación en valores a partir, por ejemplo, de la teoría del desarrollo del juicio moral, la ética del cuidado o la teoría de las emociones. Aunque tales aportaciones parciales tienen un papel indiscutible en la tarea de pensar la educación en valores, maximizar la importancia de una dimensión no resulta adecuado para entender la complejidad de la educación en valores.

¿Desde dónde pensar la educación en valores si no parece acertado emplear una estrategia fundacionalista o reduccionista? La educación en valores no parte de una verdad segura desde la cual derivar cómo debe ser la vida de cada individuo y del conjunto de la sociedad. Tampoco parte de algún saber científico que limite a alguno de sus elementos parciales la complejidad del aprendizaje de los valores. La educación en valores no necesita buscar ninguna seguridad para construirse, necesita en cambio prestar toda la atención posible a las situaciones concretas de la vida. Dicho de otro modo, debemos pensar la educación en valores desde la actualidad, preguntándonos por lo que pasa y por lo que nos pasa (Foucault, 1985). Debemos mirar el aquí y ahora, siendo sensibles a las dificultades e injusticias que plantea, pero también detectando todos aquellos aspectos de la realidad que funcionan correctamente y conviene preservar. La educación en valores comienza interrogando el mundo de la vida que nos acoge: mirando y valorando la actualidad de la vida y de nuestra vida para trazar un recorrido propio y apropiado.

\section{¿Cómo vivir?}

Dirigir la mirada hacia la actualidad es el inicio de la educación en valores porque los seres humanos estamos indeterminados, tanto en nuestra vida individual, como el modo colectivo de organizar la convivencia. Nacemos inacabados, programados solo en parte y muy plásticos y adaptables. Por otra parte, la sociedad nos recibe con un alto grado de normatividad, pero en ningún caso puede $-\mathrm{y}$ quizás tampoco quiere- borrar la capacidad de indignación, de crítica y de innovación cívica y moral. Si estamos indeterminados es precisamente para poder actuar creativamente ante las eventualidades siempre nuevas del presente. Por lo tanto, ayudados con las fuerzas de nuestra inteligencia, hemos de pensar en ir dando respuestas a una pregunta fundamental: ¿cómo vivir bien aquí y ahora?

Esta pregunta nos interroga sobre cómo pensamos que debemos vivir, dado que no está previsto en parte alguna cómo debemos hacerlo. Nos interroga sabiendo que no contamos con respuestas preparadas y que tampoco nos van a servir supuestas ideas universales, cuya simple aplicación a la realidad singular va a permitir responder a la pregunta inicial. Sin duda compartimos valores y principios morales relevantes, pero que en modo alguno nos liberan de pensar por nosotros mismos de manera libre y crea- 
tiva cómo responder a la pregunta por la forma de vida que deseamos vivir. Por otra parte, el proceso de búsqueda de respuestas moviliza las capacidades de la inteligencia moral de cada sujeto, pero también nos moviliza colectivamente al conectar cada una de las inteligencias singulares en un proceso complejo de deliberación. No tendremos una respuesta cabal sin el juego siempre individual y colectivo de la inteligencia moral.

El juego de la inteligencia moral en busca del mejor modo de vivir nos conduce a un aprendizaje que aborda las principales dimensiones de la experiencia humana: ser uno mismo, convivir, participar y habitar el mundo (Delors, 1996; Jonas, 1995; Morin, 2004). Con el reto de llegar a ser uno mismo nos referimos al trabajo que cada individuo realiza sobre sí mismo para liberarse de ciertas limitaciones, para construir una manera de ser deseada y para lograr el mayor grado posible de autonomía y de responsabilidad (Appiah, 2007; Foucault, 1990; Schmid, 2002). La experiencia del convivir apunta a la tarea que hay que llevar a cabo para superar la tendencia al propio aislamiento e individualismo, así como también la tendencia a tratar a los demás como cosas que podemos usar. Se trata de establecer vínculos basados en la apertura, la comprensión y el compromiso en la realización de proyectos comunes (Honneth, 1997; Levinas, 2000; Bello, 1997). La tarea de participar consiste en llegar a formar parte de la colectividad alcanzando un buen nivel de civismo, o respeto de las normas y hábitos públicos, y convirtiéndose en un ciudadano activo. Es decir, una persona capaz de requerir los derechos que le corresponden y al mismo tiempo sentir la obligación de cumplir los deberes y manifestar las virtudes cívicas necesarias para contribuir a la organización democrática de la convivencia (Camps y Giner, 2008; Barber, 2000). Finalmente, habitar el mundo nos propone la preocupación y el cuidado de la humanidad y de la naturaleza, del todo imprescindible en un momento en el que la globalización se extiende por todos los ámbitos de la vida y en que la crisis ecológica también se ha generalizado de manera implacable por todos los rincones del planeta (Jonas, 1995; Boff, 2001; Küng, 1991; Martínez y Hoyos, 2006).
La pregunta inicial de la educación en valores, cómo vivir hoy y aquí, nos obliga a valorar la realidad actual de todos los ámbitos de la experiencia humana. Ahí reside el punto de arranque de la reflexión moral y de la educación en valores. No partimos de seguridades, sino de la valoración de las formas de vida que nos acogen.

\section{Mapa y brújula}

Hasta aquí hemos afirmado que la educación en valores no parte de seguridades desde las que derivar normas morales, sino que parte de la valoración que a cada individuo le merece la actualidad. Una valoración que permitirá discriminar entre formas de vida que se aceptan y formas de vida que se deben cambiar. Valorar la actualidad en la que vivimos es una continua toma de posición respecto a lo que deseamos conservar y a lo que consideramos que se debe transformar. Es un proceso incesante de valoración para discernir entre las normas sociales y las costumbres morales que nos parecen aceptables, las que no consideramos acertadas -simplemente porque valoramos que son injustas- $y$, por último, para determinar los aspectos vitales sobre los que no tenemos claro cómo conducirnos. La primera tarea moral es decidir qué conductas reproducimos, qué conductas transformamos y sobre qué temáticas debemos aplicar la creatividad moral.

En cierta forma, podemos decir con Piaget (1971) que la educación moral recorre una primera etapa de heteronomía en la que los jóvenes reciben las normas de la sociedad que les acoge, para luego ir transitando hacia una segunda etapa en la que son capaces de darse a sí mismo normas morales y conducirse, por lo tanto, de manera autónoma. Es decir, pasan de un momento de inmadurez moral a otro de madurez en el cual pueden discriminar entre las normas que consideran que deben aceptar y las normas que se esfuerzan por cambiar, o incluso en detectar los ámbitos para los que es preciso inventar nuevas normas morales.

Tal como sugiere el título de este epígrafe, la educación en valores prepara para un trayecto vital que a cada paso nos interroga sobre cómo 
vivir correctamente. Pero para responder a este interrogante perpetuo contamos con dos ayudas fundamentales: el mapa y la brújula, como suele ocurrir en todos los viajes complicados (Cortina, 2001). El mapa nos indica los caminos conocidos, los caminos que se han ido marcando por el uso y el acierto. Son caminos que en principio no conviene olvidar. Pero los trayectos interesantes, como la vida, también incluyen etapas para las que no tenemos ningún mapa previo o incluso momento en los que deseamos decidir hacia donde nos vamos a dirigir. En estas situaciones debemos ayudarnos de una brújula que nos marca la dirección, aunque no el recorrido. Es el momento de dibujar un nuevo camino y, en algunos casos, decidir la dirección que deseamos tomar. Aunque la brújula nos marca el norte, quizás no es el lugar hacia el cual nos gustaría dirigirnos. En síntesis, cuando los trayectos no están claros, algo que ocurre a menudo, hará falta que cada sujeto ande su propio camino y contribuya con sus pasos a trazar una senda para los que vendrán detrás. Y en ciertos momentos hará falta tomar una dirección nueva, probarla, evaluarla $y$, en caso que los resultados sean positivos, ofrecerla a los que vengan detrás.

Si la educación en valores es una preparación para recorrer el trayecto vital en busca de un buen modo de vivir, decidiendo entre la opción heterónoma del mapa o la opción autónoma de la brújula, es imprescindible ofrecer al alumnado propuestas formativas acordes con cada una de estas dos opciones. Nadie logra aprender a conducirse moralmente sin la oportunidad de vivir experiencias formativas que refuercen la adquisición de normas morales, que inviten a su transformación o que dispongan al alumnado para la invención de nuevas posibilidades. En lo sucesivo entraremos en la justificación y desarrollo de las propuestas curriculares que nos ayudaran a alcanzar los objetivos que acabamos de formular.

\section{Prácticas morales}

De forma muy sintética podemos decir que un currículo de educación en valores es un conjunto de prácticas morales que ayudan a adquirir la competencia para valorar la actualidad y para discriminar lo que se debe aceptar y cómo adquirirlo, lo que se debe transformar y cómo cambiarlo, y lo que se debe inventar y cómo crearlo. Pero, antes de seguir, debemos aclarar ¿qué es una práctica moral?

Una práctica es un proceso con principio y final en el que participantes actúan conjuntamente en busca de un objetivo y a la vez expresan valores. Entre otros muchos ejemplos de prácticas educativas, podemos citar algunas como: las asambleas de clase, la celebración de fiestas, los momentos de intercambio entre profesor y alumno, las tareas que se realizan en las clases, la discusión de un problema y la norma que se establecen para resolverlo, los trabajos por proyectos de mayor o menor duración, por citar solo una corta relación. Son buenos ejemplos de prácticas porque muestran cómo diversos participantes actúan conjuntamente en busca de un objetivo durante un tiempo delimitado.

Aunque ya tenemos una primera aproximación al concepto de práctica, todavía podemos continuar su análisis para llegar a comprender mejor su valor educativo. Veamos algunas de sus principales características. En primer lugar, las prácticas son un curso en gran parte provisto de acontecimientos ordenados, coherentes y complejos: una sucesión de hechos. Las prácticas trazan un camino a seguir y son un surco del cual no salir. Además de una organización previsible, las prácticas presentan también cierta rutinización: tienden a repetirse con frecuencia. Que las prácticas sean cursos de acción organizados y rutinarios les da una capacidad educativa enorme porque su repetición facilita el aprendizaje. En segundo lugar, las prácticas son cursos de acontecimientos que persiguen objetivos funcionales y cristalizan valores durante su realización: son acciones moralmente informadas. Las prácticas tienen unos objetivos a los que apuntan los participantes con su acción concertada y unos valores que manifiestan durante todo el proceso. Realizamos las prácticas para obtener un resultado -sus objetivos-, y realizamos la práctica para actualizar y vivir valores. En tercer lugar, las prácticas invitan a sus participantes a adquirir virtudes. Las prácticas 
exigen que los actores alcancen niveles de excelencia acordes con los valores que expresa el curso de acontecimientos. Los participantes intentan mostrar las virtudes que exige la correcta realización de cada uno de sus pasos. Las prácticas son talleres de virtud en la medida que permiten a los actores ejercitarse y adquirir un dominio cada vez mejor de las virtudes que se requieren (Bourdieu, 1997; Giddens, 1995a; MacIntyre, 1987). En cuarto y último lugar, las prácticas se viven en situaciones de taller: una situación de aprendizaje formada por un grupo de iguales, los aprendices, y una persona que cuenta con una experiencia superior, el especialista (Schön, 1992; Rogoff, 1993). En una situación de taller, vivir una práctica provoca múltiples interacciones donde se intercambia afecto, donde se reflexiona y donde se trabaja en común a propósito del contenido concreto de cada una de las propuestas.

\section{Prácticas procedimentales y prácticas sustantivas}

Las prácticas morales tienen en común los rasgos que acabamos de exponer, pero también se diferencian en algunos aspectos importantes. Tal como pedíamos anteriormente, la educación en valores debe proporcionar experiencias de repetición moral en las que se acepta las formas dadas como buenas y también experiencias de innovación moral donde hace falta transformar las formas de vida $o$ crear otras nuevas. Las prácticas morales que debe ofrecer un currículo de educación en valores deben orientarse también en esta doble dirección. En este sentido, creemos que es posible establecer una primera distinción entre un conjunto de prácticas que llamaremos procedimentales y otro grupo al que vamos a denominar prácticas sustantivas. Con ello queremos indicar que, pese a tratarse siempre de prácticas morales, su intencionalidad puede distinguirse. Como veremos a continuación, no tiene el mismo sentido moral la realización de una asamblea o la discusión de un dilema moral que la celebración de una fiesta de cumpleaños o la evaluación de los cargos de la clase. Los primeros casos son ejemplos de prácticas procedimentales, mientras que los dos últimos se trata de prácticas sustantivas (Puig, 2003).
En el caso de las prácticas procedimentales estamos ante cursos organizados de acontecimientos que establecen un modo de conducirse que, tal como ocurre con el uso de las herramientas de trabajo o los métodos de investigación científica, nos permite buscar o crear algo moralmente valioso o correcto. Pero se trata de alcanzar algo que no se nos ofrece ya dado o acabado de antemano, sino que hemos de construir o recomponer gracias al libre uso del procedimiento moral. Las prácticas procedimentales fijan cursos de acciones que expresan valores, requieren virtudes y apuntan a finalidades morales, pero además abren un espacio a la creatividad moral y al afán de investigación moral de los sujetos. Los procedimientos morales son doblemente valiosos: lo son en sí mismos -usar bien una herramienta es una destreza que exige virtudes y cristaliza valores-, y lo son en la medida que permiten la autonomía y la invención moral -al usar una herramienta podemos producir algo nuevo $\mathrm{y}$ valioso-. Método y producto son las dos caras de las prácticas procedimentales. Cuando discutimos un dilema moral estamos usando un procedimiento basado en el diálogo como sistema para intercambiar razones - un método-, pero además su uso nos permite acercarnos a una mejor comprensión de los problemas y quizás a una solución aceptable por todos los participantes -el producto-. Las prácticas procedimentales son cursos de acontecimientos establecidos que abren un espacio a la expresión y la creatividad moral.

Por su parte, en el caso de las prácticas sustantivas estamos ante cursos organizados de acontecimientos que establecen un modo de conducirse que, tal como ocurre con el manual de instrucciones de una máquina, nos indica cada uno de los comportamientos que debemos realizar para alcanzar un fin correcto previamente fijado. Las prácticas sustantivas establecen cursos de acciones que expresan valores, requieren virtudes y apuntan a finalidades morales. Sin embargo, en el caso de las prácticas sustantivas no se abre un espacio de creatividad moral, sino un espacio de repetición moral. Las prácticas sustantivas cristalizan en comportamientos concretos y en cursos de acontecimientos establecidos 
los valores que una comunidad más desea ver realizados. Los fija y los propone para que sean adoptados por parte de los jóvenes. La celebración de una fiesta de cumpleaños establece un conjunto de pasos que exigen un cierto esfuerzo moral por parte de sus actores y que el educador va introduciendo y pautando. En estos casos se trata de reproducir la fiesta de un modo parecido cada vez que se celebra. Ciertamente se pueden introducir variaciones, y así ocurre con frecuencia, pero lo importante no es la originalidad de los pasos, ni tampoco que los puedan idear o retocar los participantes. Lo relevante de verdad es que se introduzcan en un cauce que les guíe por una senda de comportamientos valiosos y que lo haga con tal fuerza y significación que el acto de participar en la práctica adquiera un alto contenido educativo.

Para acabar, y a modo de introducción a los siguientes apartados, vamos a presentar diferentes tipos de prácticas procedimentales y de prácticas sustantivas. En lo sucesivo vamos a describir dos tipos de prácticas procedimentales, las de reflexividad y las de deliberación, y un tipo de prácticas sustantivas, las de virtud.

\section{Prácticas de deliberación}

Las prácticas de deliberación plasman un empeño arraigado en la teoría ética y también muy presente en la práctica moral: los problemas relativos al mejor modo de vivir pueden dilucidarse mediante procedimientos de indagación y argumentación racionales. La presencia de esta aspiración moral en las sociedades plurales y democráticas actuales invita a que en educación se adopten prácticas escolares de deliberación.

El esfuerzo que se pide a los individuos implicados en una práctica de deliberación es que consideren conjuntamente una cuestión que debería ser significativa y que a veces puede resultar muy controvertida. En las situaciones de deliberación interesan por supuesto las opiniones de cada uno de los alumnos, pero por encima de todo centramos la atención en el proceso de consideración de un tema y en el debate entre puntos de vista distintos. El aspecto clave es tratar una cuestión controvertida, pero hacerlo enlazando la propia opinión con la de los demás en búsqueda de una mejor comprensión de la situación analizada. En tal sentido, entendemos que deliberar significa reflexionar conjuntamente sobre una cuestión, es decir, establecer los hechos, analizar las causas que los provocan, comprender las posiciones de los implicados, analizar los motivos y las intenciones de todos ellos, valorar las consecuencias, dialogar aportando las mejores razones que pueda aducir cada uno de los puntos de vista en litigio, buscar soluciones mejores, reconocer las dificultades para encontrar puntos de acuerdo, pero reconocer también la amplitud de perspectivas que se han podido obtener durante el proceso. Un proceso que al final deberá proporcionar a todos los participantes la adquisición de conocimientos y, quizás por encima de todo, la modificación de opiniones y actitudes. Pero, en cualquier caso, se trata de una mejora que solo se alcanzará por efecto de la actividad de considerar colectivamente cuestiones significativas. Entre las prácticas deliberativas que los educadores instauran para animar la participación plena y activa de los jóvenes en procesos dialógicos encontramos estrategias tan variadas como los ejercicios de comprensión crítica (Puig, 1995, pp. 185-210), orientados a cuestionar la realidad, los programas de filosofía para niños creados para entrenar el razonamiento y la crítica en contextos de diálogo (Lipman, 1988) o las actividades que parten de la literatura y el cine como elementos de reflexión y discusión (Rorty, 1991). A continuación mostraremos dos de las propuestas educativas que, quizás, de manera más clara contemplan el diálogo como herramienta especialmente eficaz para abordar temas controvertidos de interés para el grupo: la asamblea escolar y la discusión de dilemas morales.

Entre las prácticas educativas que, de manera más clara, cumplen con los elementos que hemos presentado, encontramos la asamblea escolar y la discusión de dilemas morales, dos propuestas que contemplan el diálogo como herramienta especialmente eficaz para abordar temas controvertidos de interés para el grupo.

\section{La asamblea de clase}

La asamblea escolar tiene como finalidad la participación de los miembros del grupo mediante el 
uso de la palabra. Es el momento institucional del diálogo: una reunión periódica que se destina de manera exclusiva a que alumnos y educadores conversen de todo aquello que les parezca oportuno en orden a optimizar el trabajo y la convivencia. Un espacio para dialogar con ánimo de entenderse, organizarse mejor y solucionar los conflictos de relación que puedan plantearse.

La práctica de la asamblea parte del supuesto de que los jóvenes son capaces de reflexionar sobre sí mismos, tomar conciencia de su realidad grupal y llegar a acuerdos que les permitan resolver sus problemas. En la asamblea no hay soluciones determinadas a priori ni el grupo está sometido a una autoridad exterior, tampoco dispone de un código moral al que obedecer. Por el contrario, a partir de las distintas singularidades y contrastando puntos de vista e intereses diversos, los jóvenes deliberan con la voluntad de encontrar una solución justa a la situación que se han planteado. Las habilidades dialógicas son un elemento clave en esta actividad. En la medida que los jóvenes adquieren un buen dominio de ellas se optimiza el proceso y los resultados de la asamblea, Sin embargo, un dominio pobre del diálogo no invalida la práctica de la asamblea. Las habilidades para el diálogo se adquieren mediante el ejercicio continuado y la asamblea cumple también una función formativa, dando la oportunidad a los jóvenes de aprender y mejorar sus competencias dialógicas.

En cuanto práctica educativa, la asamblea propone una secuencia de fases, cada una de las cuales exige a quienes participan, determinados comportamientos. A continuación presentaremos el protocolo de esta práctica, entendiendo que deberá adaptarse a cada contexto, pero que en sí mismo apunta a horizontes de valor y predispone a la adquisición de virtudes (Puig, Martín, Escardíbul y Novella, 1999).

El primer paso de una asamblea es la preparación. Las cuestiones que se discuten en la asamblea las propone el grupo y serán los alumnos encargados de conducirla junto con el tutor quienes establezcan los temas para el debate. La convivencia y el trabajo son ámbitos que implican a la colectividad y es responsa- bilidad de todos que funcionen de manera adecuada. Cada miembro del grupo está legitimado para plantear aquello que considere relevante y confía en que sus compañeros tendrán en cuenta sus propuestas.

El debate es el segundo momento de la asamblea y constituye el núcleo de esta práctica. En él se produce un intercambio de opiniones y propuestas sobre un tema, orientadas a alcanzar una solución satisfactoria para todos. Pero la asamblea no busca acuerdos precipitados. Es un espacio destinado a hablar y escuchar hasta acercarse al entendimiento. Previamente al logro de un acuerdo los jóvenes deben argumentar la propia opinión, escuchar las distintas posturas, esforzarse por comprenderlas y estar dispuestos a ceder. Mientras entrenan los comportamientos que el debate les exige, adquieren las virtudes que sustentan su conducta. El debate supone siempre un ejercicio de asociación que se desarrolla a partir del contraste entre los diferentes puntos de partida e intereses que conviven en el grupo.

El tercer momento de la asamblea, la aplicación, tiene como objetivo ayudar a que los acuerdos se traduzcan en la vida cotidiana del grupo. La discusión en asamblea de los temas que se proponen predispone y compromete a los participantes a cumplir lo que han acordado. En esta fase los jóvenes deben aplicar las soluciones que juntos han formulado para resolver sus problemas, sabiendo que no se trata de soluciones definitivas sino de acuerdos revisables que serán sometidos a la crítica en posteriores debates, y se modificarán cuando el grupo lo consideré oportuno.

La participación repetida en esta práctica deliberativa, la asamblea escolar, refuerza el reconocimiento de la riqueza que pueden aportar los puntos de vista diferentes ante una misma situación y permite a los jóvenes sentirse parte solidaria de un grupo a través del ejercicio de la deliberación. Algunos centros de secundaria optan por implementar la mediación escolar como una práctica alternativa a la asamblea, priorizando la participación de los jóvenes en temas vinculados a la convivencia en detrimento de cuestiones organizativas y de trabajo. 


\section{Discusión de dilemas morales}

La discusión de dilemas morales es una estrategia que tiene como eje central el diálogo aplicado a situaciones controvertidas. A partir de una breve narración se presenta una disyuntiva de valores difícil de resolver ya que exige optar entre valores en alguna medida deseables. El hecho de que los dilemas planteen alternativas contrapuestas que permiten ser defendidas con argumentos legítimos complica el posicionamiento a favor o en contra de cada una de las opciones a la vez que estimula en los participantes la reflexión, la producción de argumentos que justifiquen sus opiniones, así como la valoración crítica de los razonamientos propios y ajenos. En tanto que metodología, la discusión de dilemas morales se orienta al desarrollo del juicio moral individual, capacidad que ayuda a elaborar argumentos respecto a la corrección o incorrección de los hechos morales (Kohlberg, 1992). Si bien se trata de un procedimiento innato de carácter universal que se ejercita continuamente, conseguir un nivel óptimo de juicio moral es especialmente útil cuando la persona se enfrenta a situaciones conflictivas en las que resulta difícil tomar una decisión. Aun tratándose de una capacidad innata, el contexto ejerce un papel importante a la hora de estimular, posibilitar o dificultar su desarrollo, hecho que tiene consecuencias en el ámbito de la intervención educativa. Así, las escuelas que ofrecen oportunidades para practicar la capacidad de argumentación en relación a temas controvertidos, favorecen en sus alumnos el avance hacia niveles superiores de razonamiento moral, mientras que aquellas instituciones que no permiten el debate estarían obstaculizando el desarrollo natural del juicio moral. A nivel didáctico los dilemas morales exigen la presencia de una serie de elementos que facilitan la percepción individual del conflicto y la discusión en grupo: una situación que muestra un conflicto moral, un protagonista que asume la responsabilidad de decidir, dos alternativas que plantean una disyuntiva de valor, y una pregunta que exige el posicionamiento a cada uno de los participantes en la discusión. Además, con frecuencia, se incluyen otros interrogantes que modifican algún elemento del dilema inicial, creando nuevas controversias y dilemas alternativos.
La estimulación hacia estadios superiores de juicio moral, finalidad prioritaria de la discusión de dilemas morales, requiere, según los psicólogos del desarrollo (Hersh, Reimer y Paolitto, 1984) de una experiencia interna de conflicto cognitivo que rompa la seguridad de los propios razonamientos y fuerce al sujeto a buscar nuevos criterios de argumentación que le permitan solucionar el conflicto y le devuelvan la seguridad en el propio juicio moral. Aunque el dilema moral está pensado para favorecer situaciones de desequilibrio interno, no siempre el sujeto reconoce como propio el problema que se le plantea. La discusión en grupo y el debate ahondan en esta cuestión ayudando a los participantes a percibir la controversia o el desacuerdo moral que encierra el dilema. La heterogeneidad de puntos de vista, intereses y perspectivas de las diversas aportaciones constituyen un soporte inapreciable para el desarrollo moral. A partir del intercambio dialógico cada miembro del grupo defiende sus puntos de vista basándose en argumentos racionales que puedan ser rebatidos y discutidos por el resto de interlocutores. Aunque la finalidad de la discusión no es llegar a un acuerdo, es interesante encaminar el diálogo a acercar posturas enfrentadas y a reconocer los aspectos compartidos o que se han clarificado a lo largo del proceso deliberativo. Pese a que la esencia de esta práctica consiste en discutir sobre temas significativos que presenten una controversia de valores, la discusión de dilemas morales propone una trayectoria metodológica concreta que favorezca el logro de sus objetivos. La discusión se inicia a partir de un primer contacto del grupo con el dilema, ya sea a partir de la lectura individual o la presentación del texto por parte del profesor; se continúa con una toma de postura individual, que conviene realizar de forma escrita, y que prepara para el siguiente paso, la discusión del dilema en grupos reducidos, en donde cada participante argumenta su opinión y la contrasta con los puntos de vista de sus compañeros. Por último, se realiza una puesta en común del trabajo realizado en los grupos. Una vez concluido el debate también se puede pedir a los alumnos que vuelvan a posicionarse por escrito ante el dilema. En este segundo escrito suelen quedar reflejadas 
las modificaciones que cada miembro del grupo ha introducido en su argumentación a partir de la exposición a puntos de vista diversos, que se han mostrado en las aportaciones de sus compañeros.

La discusión de dilemas morales es una práctica especialmente significativa de deliberación, ya que no solo permite la diversidad de opiniones ante una situación en la que aparece una confrontación de valores, sino que además alienta el disenso y crea las condiciones idóneas para que el grupo analice la situación, reflexione sobre los pros y contras de cada alternativa, valore sus consecuencias y, sobre todo, se comprometa a buscar maneras de resolver el conflicto considerando las mejores razones aportadas desde los distintos posicionamientos.

\section{Prácticas de reflexividad}

Las prácticas de reflexividad expresan una de las aspiraciones éticas más antiguas del pensamiento de Occidente: el conocimiento y el cuidado de sí mismo. El trabajo sobre sí mismo permitió gestar la idea de individualidad, una idea que más adelante culminó en la aspiración moderna a la plena autenticidad y autorrealización personal. Tras múltiples cambios y mutaciones, el autoconocimiento, la sinceridad con uno mismo, la autoestima, la autoevaluación y la autorregulación se han convertido en las formas más representativas que toma en la actualidad el ideal ético de la reflexividad. La presencia generalizada de este horizonte ético en la conciencia moderna convierte en una tarea relativamente sencilla el encontrar en nuestras escuelas prácticas que realicen las aspiraciones éticas de reflexividad y autenticidad.

Este tipo de práctica en sus distintas modalidades invita a cada individuo a mirarse a sí mismo a propósito de alguna cuestión relevante. Pero la intención no es tanto indagar en el tema que centra la atención, como indagar en el propio sujeto a propósito del tema en cuestión. Es un esfuerzo de introspección por reconocer cuál es la opinión, cuáles los sentimientos, cuál la posición o simplemente la información que el sujeto puede producir en relación con la temática que se está tratando. Estamos ante una indagación sobre el yo de cada individuo que se realiza a través de la mediación que introduce el tema que centra su atención. Para conseguir estos objetivos las escuelas disponen de múltiples ejercicios y estrategias que estimulan procesos de autorreflexión, como los ejercicios de autoconocimiento y la autobiografía guiada.

\section{Conocimiento de uno mismo}

Los ejercicios de conocimiento de uno mismo incluyen distintas técnicas y estrategias formativas cuya finalidad es ayudar al sujeto a adoptar de manera progresiva un sistema de valores cada vez más definido que le permita conducirse a sí mismo. La autonomía personal está en el horizonte de estas propuestas y para lograrla es necesario el ejercicio reflexivo orientado a la toma de conciencia de los valores que dominan en las distintas áreas de la vida y al esfuerzo personal por convertirlos en guías para la acción.

Ante la pluralidad de propuestas morales y éticas que caracterizan las sociedades plurales es imprescindible, desde el ámbito educativo, dotar a los jóvenes de herramientas que les ayuden a construir su propia jerarquía de valores. Una tarea necesaria porque los valores se definen como reguladores que marcan los objetivos que persigue la acción humana y la modifican para dirigirla hacia los objetivos deseados (Raths, Harmin y Simon, 1967). Pero además de guiar la conducta los valores también dan sentido de un modo más global a la biografía de cada sujeto. Los ejercicios de clarificación de los valores, orientados al autoconocimiento y la autorregulación, apuestan por un proceso de clarificación orientado, al menos, a tres mecanismos de adquisición de valores. En primer lugar, se adquieren valores gracias a la toma de conciencia de lo que realmente se prefiere. Frente al desconocimiento, la clarificación es un proceso de mirada interior que detecta cuáles son los verdaderos valores, los que realmente se desean para uno mismo. En segundo lugar, se da un proceso de elección de valores cuando la persona es libre de escoger entre distintas alternativas. Clarificar valores exige también un ejercicio de reconstrucción personal que supone el redescubrimiento o la creación de valores y de maneras de aplicarlos a la 
situación concreta en que se encuentra cada sujeto. Pero además de la elección reflexiva, un proceso de clarificación incorpora el aumento del aprecio por los valores elegidos que lleva a considerarlos parte importante de la propia manera de ser y que, llegado el caso, invita a defenderlos. Asimismo la adquisición de valores culmina en la capacidad de actuar de acuerdo con ellos. Es decir, de esforzarse para que la conducta sea el reflejo de los valores adquiridos y se repita hasta convertirla en un hábito.

Dado que los procesos de autoconocimento apuntan a valores y preferencias que se manifiestan en múltiples aspectos de la vida personal, los ejercicios suelen abordar las aspiraciones personales, los temas que cada sujeto acaba encontrando a lo largo de su biografía -estudios, amistad, amor, trabajo, sexualidad, religión, etc.-, y también suelen plantearse temas sociales relevantes desde el punto de vista personal o colectivo. En cualquier caso, todos los temas que afecten a la persona pueden ser objeto de un esfuerzo de autoobservación clarificación y autorregulación que intentará establecer los fines, aspiraciones, intereses, actitudes, sentimientos, y convicciones que cada sujeto detecta en sí mismo a propósito de la cuestión considerada.

Los ejercicios orientados al conocimiento de uno mismo pueden ser muchos y muy variados, pero quizás merece la pena citar el antecedente de todos ellos: las preguntas socráticas. Mediante un conjunto de preguntas Sócrates pretendía, primero, mostrar que en realidad su interlocutor estaba bastante confundido sobre el tema sometido a debate y, luego, ayudarle a sacar de sí mismo todo lo que en realidad sabía y hasta ese momento había quedado oscurecido. Aprender era descubrir o iluminar lo ya sabido: clarificar el propio pensamiento. En la actualidad hay un importante número de estrategias didácticas deudoras de las preguntas socráticas, entre las más conocidas encontramos los ejercicios de frases inacabadas y los ejercicios expresivos. En los ejercicios de frases inacabadas se invita al alumnado a responder a un conjunto de preguntas y frases inconclusas sobre un tema significativo, lo que les exige aclarar su punto de vista respecto a cada una de las cuestiones planteadas. El trabajo individual puede continuarse con la realización de un debate colectivo sobre algunos de los aspectos abordados. Los ejercicios expresivos incluyen un conjunto de dinámicas que sirven para que el alumno descubra y manifieste algún sentimiento, experiencia, pensamiento o valor. En muchos casos estos ejercicios tienen una intención netamente indagadora o de autoanálisis. Los ejercicios expresivos más corrientes son los escritos de todo tipo -redacciones, poesías o comentarios-, los dibujos, las representaciones, los ejercicios de mímica, y los trabajos audiovisuales. Mediante todos ellos, convenientemente adaptados a la edad del grupo, pueden obtenerse importantes logros en el proceso de autoconocimiento y expresión de sí mismo. En otros casos, los ejercicios expresivos tienen, más que una voluntad estética, una intención netamente indagadora o de autoanálisis.

\section{Autobiografías guiadas}

Si la clarificación de valores atiende a la toma de conciencia de los propios valores, los ejercicios autobiográficos se orientan hacia la elaboración de la identidad personal en tanto que historia de la propia experiencia vital (Giddens, 1995b). Se trata no solo de asumir valores, sino también de integrarlos en una historia personal a la que den sentido. El objetivo es que la persona vaya reconociendo, asumiendo y dando sentido a su pasado y vaya, asimismo, construyendo un horizonte de futuro que le permita seguir integrando su experiencia. Ambas condiciones, entender el propio pasado y proyectar el futuro, apelan a la práctica reflexiva y son fundamentales en la formación de una personalidad autónoma.

La palabra autobiografía engloba aquello que pretende decir. Auto viene a significar el yo consciente de sí mismo; bios se refiere a la continuidad histórica y vital de esa identidad; y grafía designa el medio técnico de la escritura autorreferencial. A menudo se critica la escritura autobiográfica argumentando que el lenguaje no puede reflejar realmente la vida vivida. Sin embargo, las posibles limitaciones de la escritura se mitigan cuando la autobiografía es leída y cuando el lector devuelve al autor la interpretación de lo leído y de este modo 
ambos traban un diálogo a propósito del texto que les permitirá mejorar su comprensión. El texto y el diálogo pueden ser para su autor un soporte o anticipación de su proceso de construcción personal. Una modalidad de redacción y lectura de textos autobiográficos que puede aplicarse al ámbito educativo son los grupos de trabajo para la redacción de autobiografías guiadas. Se trata de prácticas que combinan la redacción personal de textos autobiográficos con su lectura y comentario en grupo (Martín, Puig, Padrós, Rubio y Trilla, 2003) con la finalidad de facilitar a los participantes una experiencia de comprensión crítica de sí mismos. Es decir, una experiencia que les permita enfrentarse a su vida desde las voces que comparten su mente, de modo que la rememorización vaya acompañada del diálogo interno que les ayudará a valorar su experiencia. Posteriormente, se enfrentarán al escrito sobre su vida con la ayuda de las producciones de los demás participantes y del diálogo que construyan. Estos dos momentos facilitan un trabajo sobre el pasado para explorarlo, reconocerlo y organizarlo, un trabajo de reconceptualización de sí mismo que debería suponer la integración de lo rememorado, y un trabajo de proyección hacia el futuro. Estos pasos tienden a dar mayor sentido y valor a la propia existencia, lo cual en realidad es la finalidad última de la práctica. Las tareas concretas que supone la participación en un grupo de trabajo, son fundamentalmente de tres tipos: promover la autoconciencia mediante la redacción de un texto que obligue al sujeto a pensar reflexivamente sobre su pasado, su presente y su futuro; promover la abertura que permita compartir con su grupo la experiencia que acaba de escribir; y promover una visión alternativa de la biografía personal de modo que puedan reinterpretarse las experiencias del pasado del modo más favorable posible sin por ello perder la veracidad.

Respecto a la dinámica seguida en los grupos para la redacción de autobiografías suele planificarse de acuerdo con las siguientes características. A ser posible se forma un grupo de unas diez personas con el que se realizan unas ocho o nueve sesiones. El educador organiza las tareas y decide los temas sobre los que se escribirá, crea un clima de grupo cálido que facilite la abertura de sus miembros, conduce el trabajo en grupo, y evita cualquier conducta de imposición o de coacción. Un buen clima y la ausencia de presión son las principales condiciones para desinhibir la lectura. Los temas sobre los que se puede escribir los proponen los educadores apoyándose si lo consideran oportuno en las sugerencias de los participantes. La estructura de las sesiones contempla un tiempo destinado a la información sobre la escritura autobiográfica y su aplicación como método formativo. En principio cada sesión se inicia comentando las dificultades que se han encontrado en el proceso de escritura y se continúa con la lectura de las distintas producciones, tarea que se realiza primero por parejas, a fin de facilitar la dinámica. En cualquier caso, se advierte que si alguien no quiere leer no tiene por qué hacerlo. Una vez terminada esta fase se repite, pero ahora con todos los participantes juntos. La lectura de todos los textos deriva en diálogos sobre temas diversos, referentes, en su mayoría, al contenido de lo leído pero otras a temas tan solo relacionados con lo tratado. Finalmente, de vez en cuando se intenta obtener algunos comentarios valorativos para reorientar en la medida de lo posible la marcha de la experiencia.

\section{Prácticas de virtud}

Lentamente va abriéndose paso de nuevo la reflexión sobre las virtudes. Hablar sobre cualidades admirables del carácter, hablar sobre hábitos de conducta deseados, hablar, en fin, sobre formas de excelencia a las que deben aspirar los humanos, no parece ya una cuestión del pasado. En la actualidad, los educadores saben muy bien qué rasgos de carácter son apreciables y, a poco que se lo propongan, pueden establecer un listado de las cualidades personales que desearían transmitir a sus alumnos. Se hable o no de virtudes no parece que estemos muy lejos de ellas. Las prácticas de virtud cubren necesidades escolares, cristalizan valores y, además, se despliegan gracias a la participación de cada sujeto en un curso de acciones conjuntas e intercambios lingüísticos con los demás implicados. Las prácticas de virtud suponen hacer y hablar para cumplir con 
una función formativa. Son un entramado de comportamientos activos y comunicativos a través de los cuales se viven los valores inscritos en los distintos momentos de la realización de la práctica. En relación al tema de los valores, las prácticas de virtud persiguen según los casos valores distintos. Es como si cada práctica tuviese algunos valores predominantes. En cierto modo es así, pero también es verdad que todas las prácticas amalgaman una variedad de valores que hace muy difícil establecer precisamente uno o unos pocos valores básicos. La tutoría entre iguales, el aprendizaje cooperativo (Pujolas, 2008), la confección de grupos de trabajo, el trabajo por proyectos (Martín, 2006) o el aprendizaje servicio, muestran cómo además de los valores que cada una de ellas persigue de manera especialmente intensa, también aparecen otros muchos que les dan forma e identidad y que incrementan su potencial formativo. Las dos prácticas que hemos elegido para ilustrar las prácticas de virtud, la tutoría entre iguales y el aprendizaje servicio, comparten la voluntad de estimular la responsabilidad y el compromiso de los alumnos. Ambas juegan con dos elementos comunes: aprendizajes académicos y actividad. Mientras que la tutoría entre iguales centra su interés en el contexto escolar y las relaciones entre compañeros, el aprendizaje servicio extiende el compromiso más allá de la escuela y ofrece a los alumnos la posibilidad de implicarse en la mejora social.

\section{La tutoría entre iguales}

La práctica de la tutoría entre iguales es una modalidad de aprendizaje cooperativo que parte de la potencialidad que los compañeros tienen para enseñar. Se define como un sistema de instrucción formado por una pareja de alumnos, en la cual uno de los miembros enseña al otro a solucionar un problema, completar una tarea, aprender una estrategia, o dominar un procedimiento, dentro de un marco planificado exteriormente (Fernández y Melero, 1995). Habitualmente es el maestro quien organiza las tareas y la situación interactiva y también quien asume la responsabilidad, en último extremo de los aprendizajes.

Las experiencias escolares de tutoría entre iguales son muy variadas si bien existe una serie de carac- terísticas comunes que permiten discriminar qué actividades se consideran tutoría entre iguales y cuáles no. En primer lugar, se requiere la creación de un contexto de enseñanza aprendizaje en la que los alumnos implicados tienen clara conciencia de la situación en la que participan. La relación entre alumno tutor y alumno tutorado está definida por comportamientos de ayuda, apoyo y guía. Para que la práctica funcione son necesarias relaciones asimétricas en la pareja respecto al objetivo que se persigue. Es decir, que uno de los miembros -habitualmente aquel que posee mayores conocimientos en relación al aprendizaje que se debe adquirir- asume una mayor responsabilidad. Por último, se requiere la existencia de una meta definida previamente que debe conseguirse dentro de la relación de tutoría y que permite a los alumnos iniciar el proceso de enseñanza aprendizaje.

Pese a que esta práctica se inicia con la creación de parejas de compañeros, la tutoría entre iguales no consiste únicamente en juntar a dos alumnos. Su efectividad, que ha sido avalada por múltiples investigaciones, se relaciona directamente con la existencia de determinadas condiciones que garanticen la calidad de la interacción. Dado que su éxito académico depende, en parte, de la preparación que tienen los alumnos responsables del aprendizaje de su compañero, la formación de los tutores es un elemento clave para conseguir logros significativos. Asimismo un alto dominio por parte los alumnos tutores del contenido a enseñar mejora su rendimiento y les ayuda a usar una mayor variedad de recursos en el ejercicio de su rol, facilitando de esta manera las posibilidades de aprendizaje de sus compañeros. Pero el buen dominio de los contenidos no garantiza buenas prácticas de enseñanza. Una estrategia de gran utilidad para el alumno tutor es disponer de pautas concretas y bien estructuradas que guíen su intervención y le den confianza a la hora de desarrollar su tarea. También le ayuda a aumentar su nivel de rendimiento el hecho de mantener el rol de tutor durante un margen amplio de tiempo que le permita consolidar la adquisición de estrategias para ayudar a su compañero. Y finalmente un elemento que estimula la tarea del alumno tutor a lo largo del proceso es recibir información del pro- 
greso de su compañero. Saber que lo está haciendo bien es un estímulo para continuar implicándose y esforzándose en conseguir buenos resultados (De la Cerda, 2013).

Si bien la tutoría entre iguales en la escuela ha sido aplicada a situaciones diversas, vale la pena subrayar el éxito generalizado del uso de esta estrategia en el acompañamiento a la lectura y en el aprendizaje de cálculo matemático. Son muchas las escuelas que han optado por implicar a los alumnos mayores en el aprendizaje de la lectura o en el desarrollo de habilidades de cálculo de compañeros de clases inferiores. En ambos casos, la intervención de los maestros de los más pequeños a la hora de planificar las tareas así como la formación que han impartido a los alumnos mayores son factores claves de la eficacia de la intervención. Sin embargo, la diferencia de edad no es un requisito indispensable de la tutoría entre iguales. De hecho, son muchos los docentes que aprovechan esta estrategia de aprendizaje académico en el interior de sus aulas, formando díadas entre alumnos de la misma edad.

\section{Aprendizaje servicio}

El aprendizaje servicio es una práctica de virtud que busca implicar a los jóvenes en el bien común a partir de la adquisición de aprendizajes de contenidos, valores y estrategias. Se trata de una propuesta educativa que combina procesos de aprendizaje y de servicio a la comunidad en un solo proyecto bien articulado (Puig, Batlle, Bosch y Palos, 2006). Una metodología que al unir aprendizaje y servicio transforma y añade valor a ambos, y crea incluso nuevos e inesperados efectos positivos. Sobre un tema de relieve, los jóvenes estudian, investigan, reciben lecciones y, en definitiva, adquieren conocimientos curriculares. Y, a su vez, sobre esta misma temática, proyectan una actividad de servicio a la comunidad, usan lo que han aprendido, se organizan para realizarla, la llevan a cabo, y obtienen experiencia, conocimientos y nuevos interrogantes que a su vez podrán investigar de nuevo en clase. Es decir, participan en tanto que ciudadanos en la vida de su comunidad y aprenden en tanto que alumnos sobre todo ello en la escuela y durante el mismo servicio. Pero pongamos un ejemplo. Cuando los alumnos reciben en sus clases información sobre situaciones de marginación, aprenden de qué modo pueden prestar ayuda y, finalmente, colaboran con diversas entidades en las tareas asistenciales que realizan habitualmente, se está proporcionando a los jóvenes una experiencia de relación, sensibilización $y$ ayuda de un gran potencial formativo; una experiencia que les permite adquirir aprendizajes mientras preparan y realizan una tarea de servicio referida a una necesidad social.

El aprendizaje servicio es una práctica compleja porque combina varios componentes, entre los que destacan tres: necesidades sociales, aprendizajes y servicio (Puig, 2009). Y es también una propuesta contextual porque gran parte de la actividad se realiza en el contexto real, en el mismo lugar donde se manifiesta la necesidad social que el grupo trata de paliar. El aprendizaje servicio comienza con un ejercicio de análisis crítico de la realidad que permite detectar las necesidades sociales de la comunidad y determinar qué tipo de intervención se puede realizar para mejorar aquello que se considera incorrecto o deficitario. Este es el caso de un grupo de alumnos que realizan una encuesta para ver qué tipo de vida llevan los ancianos del barrio y que necesidades experimentan. Es probable que detecten deficiencias que ellos no pueden paliar, pero también es probable que puedan comprometerse en la mejora de algún aspecto concreto. Pero la detección de necesidades no es suficiente. La práctica de aprendizaje servicio pretende implicar a los jóvenes en la realización de un servicio a la comunidad. Es decir, una acción real, que va más allá de las palabras o las intenciones, y que se centra en la realización de una contribución a la comunidad, alejándose todo lo posible del asistencialismo y buscando que entre quien da y quien recibe la ayuda se cree un lazo de afecto y de reciprocidad.

Es evidente que los ámbitos que permiten acciones de servicio en beneficio de la comunidad son diversos. Citamos algunos a modo de ejemplos: experiencias de intercambio generacional, propuestas de defensa del medio ambiente, actividades de participación ciudadana, intervenciones en los medios de comunicación, defensa del patrimonio cultural, proyectos de solidaridad y cooperación, planes de promoción de la salud y ayuda educativa 
a los iguales. A pesar de la importancia de la detección de necesidades y del servicio a la comunidad, una actividad de aprendizaje servicio incorpora una fuerte preocupación por vincular ambos elementos al aprendizaje escolar que prescriben los currículos. Las maneras de llevar a cabo la conexión entre servicio y aprendizaje pueden ser variadas. A veces, se parte de un contenido curricular para buscar el modo de relacionarlo con alguna necesidad social. Un estudio de botánica propio de naturales puede acabar en una intervención en algunas zonas ajardinadas de la ciudad. En otros casos, se parte de una posibilidad de servicio que se deberá relacionar con las materias escolares. La propuesta de un banco de sangre para que los alumnos impulsen una campaña de donación en su barrio, permite estudiar aspectos científicos relacionados con el sistema circulatorio y las transfusiones, o bien tratar contenidos conectados con la comunicación y la publicidad. De un modo u otro, servicio y aprendizaje deben encontrar alguna forma de retroalimentarse.

Las finalidades que orientan la práctica del aprendizaje servicio apuntan con claridad a la adquisición de valores y de virtudes. El aprendizaje servicio pretende formar ciudadanos en la medida que impulsa la participación informada, responsable, y activa para realizar proyectos que buscan el bien del conjunto de la sociedad. Educar en valores, dado que pretende enseñar valores ampliamente compartidos a través de su puesta en práctica en situaciones reales; y finalmente, dar sentido al saber escolar, ya que se trata de adquirir conocimientos para aplicarlos y mejorar las condiciones de vida de todos, y además adquirirlos distanciándose del verbalismo en beneficio de formas de investigación basadas en la actividad de los jóvenes (Dewey, 1967).

\section{Hacia una cultura moral plural y compleja}

De lo mostrado en las páginas anteriores, pueden concluirse tres ideas principales. En primer lugar, la educación en valores no parte ni busca seguridades que de un modo cierto indiquen cómo se debe vivir, sino que se esfuerza por anclar su reflexión en la consideración del presente, en una mirada crítica a la actualidad. Una mirada que deberá esclarecer lo que se debe conservar y lo que conviene transformar $y$, en consecuencia, que nos lanzará a un esfuerzo individual y colectivo por encontrar nuevos caminos que nos ayuden a vivir de un modo más justo, solidario y feliz.

En segundo lugar, el escrito ha querido mostrar la necesidad de abrazar una reflexión ética plural que nos conduzca hasta una educación en valores también plural. Es decir, para enfrentarse a los distintos tipos de situaciones y problemas que nos plantea la realidad no basta una ética en particular, por relevante que nos pueda parecer, sino que muy probablemente deberemos usar de acuerdo a las circunstancias múltiples reflexiones éticas y recomendaciones morales. De este modo, la misma educación en valores se convierte en una reflexión plural que utiliza y se vale en cada momento de todas aquellas ideas que le ayudan a resolver los problemas con que se enfrenta. La educación en valores no es una cuestión de adscripción teórica, sino de reflexión y de acción para que todos los seres humanos consigan vivir una vida digna y mejor.

En tercer lugar, un currículo de educación en valores tampoco puede circunscribirse a una o unas pocas propuestas, aunque nos parezcan relevantes y bien argumentadas. La educación en valores requiere poner en funcionamiento en cada centro y en cada aula escolar un sistema de prácticas morales que desde diversos ángulos y de muchos modos inviten a vivir valores y logren paso a paso convertirlos en virtudes y disposiciones personales. Un conjunto de prácticas que acaban constituyendo un sistema que se retroalimenta y complementa consiguiendo de este modo una mayor influencia en los jóvenes. En definitiva, creando una cultura moral que se manifiesta en todo aquello que se hace, se habla y se siente en los centros educativos. La complejidad, que no complicación, de la educación en valores crea una atmósfera educativa que termina por calar en el modo de ser de nuestro alumnado.

\section{Referencias bibliográficas}

Appiah, K.A. (2007). La ética de la identidad. Buenos Aires: Katz. 
Barber, B.R. (2000). Un lugar para todos. Barcelona: Paidós.

Bello, G. (1997). La construcción ética del otro. Oviedo: Nobel.

Boff, L. (2001). Ética planetaria desde el Gran Sur. Madrid: Trotta.

Bourdieu, P. (1997). Razones prácticas. Barcelona: Anagrama.

Camps, V. y Giner, S. (2008). Manual de civismo. 6a. ed. Barcelona: Ariel.

Carr, W. (2007). Educación sin teoría. En: W. Carr. El docente investigador en educación (pp. 49-88). México: UNICACH.

Cortina, A. (2001). Contracte i aliança. Barcelona: Cruilla.

De la Cerda, M. (2013). Por una pedagogía de ayuda entre iguales. Barcelona: Graó.

Delors, J. (1996). Educació: hi ha un tresoramagat a dins. Barcelona: Centre UNESCO de Catalunya.

Dewey, J. (1967). Experiencia y educación. Buenos Aires: Losada.

Fernández, P. y Melero, M. A. (1995). La interacción social en contextos educativos. Madrid: Siglo XXI.

Foucault, M. (1985): ¿Qué es la Ilustración? En: M. Foucault. Saber y verdad (pp. 197-207). Madrid: Ediciones de la Piqueta.

Foucault, M. (1990). Tecnologías del yo. Barcelona: Paidós/ICE-UAB.

Giddens, A. (1995a). La constitución de la sociedad. Buenos Aires: Amorrortu.

Giddens, A. (1995b). Modernidad e identidad del yo. Barcelona: Península.

Hersh, R.; Reimer, J. y Paolitto, D. (1984). El crecimiento moral de Piaget a Kohlberg. Madrid: Narcea.

Honneth, A. (1997). La lucha por el reconocimiento. Barcelona: Crítica.

Jonas, H. (1995). El principio de responsabilidad. Barcelona: Herder

Jonas, H. (2004). El principio de responsabilidad. Ensayo de una ética para la civilización tecnológica. Barcelona: Herder.

Kohlberg, L. (1992). Psicología del desarrollo moral. Bilbao: Desclée de Brouwer.

Küng, H. (1991). Proyecto de una ética mundial. Madrid: Trotta.

Levinas, E. (2000). Ética e infinito. Madrid: Machado Libros.
Lipman, M. (1988) Investigación ética. (Manual del profesor para usar con Lisa). Madrid: Ediciones de la Torre.

Lyotard, J.F. (1979). La condition postmoderne: rapport sur le savoir. París: Minuit.

MacIntyre, A. (1987). Tras la virtud. Barcelona: Crítica.

Martín, X.; Puig, J.; Padrós, M.; Rubio, L. y Trilla, J. (2003). Tutoría. Técnicas, recursos y actividades. Madrid: Alianza.

Martín, X. (2006). Investigar y aprender. Como organitzar un proyecto. Barcelona: Horsori.

Martínez, M. y Hoyos, G. (coord.) (2006). La formación en valores en sociedades democráticas. Barcelona: Octaedro.

Morin, E. (2004). La méthode 6. Éthique. París: Seuil.

Piaget, J. (1971). El criterio moral en el niño. Barcelona: Fontanella.

Puig, J. (1995). La educación moral en la enseñanza obligatoria. Barcelona: Horsori.

1999). Feina d'educar. Relats sobre el dia a dia d'una escola. Barcelona: ICE-Horsori.

Puig, J. (2003). Prácticas morales. Barcelona: Paidós.

Puig, J. (coord.) (2009). Aprendizaje servicio (ApS). Educación y compromiso cívico. Barcelona: Graó.

Puig, J.; Martín, X.; Escardíbul, S. y Novella, A. (1999). Cómo fomentar la participación en la escuela. Barcelona: Graó.

Puig, J.; Batlle, R.; Bosch, C. y Palos, J. (2006). Aprendizaje-servicio. Educar para la ciudadanía. Barcelona: Octaedro.

Pujolás, P. (2008). 9 ideas clave. El aprendizaje cooperativo. Barcelona: Graó.

Putnam, H. (2013). Ética sin ontología. Barcelona: AlphaDecay.

Raths, L.; Harmin, M. y Simon, S. (1967). El sentido de los valores en la enseñanza. México: Uthea.

Rogoff, B. (1993). Aprendices del pensamiento. Barcelona: Paidós.

Rorty, R. (1991). Contingencia, ironía y solidaridad. Barcelona: Paidós.

Schmid, W. (2002). En busca de un nuevo arte de vivir. Valencia: Pre-Textos.

Schön, D.A. (1992). La formación de profesionales reflexivos. Barcelona: Paidós.

Stenhouse, L. (1984). Investigación y desarrollo del curriculum. Madrid: Morata.

Vattimo, G. (2010). Adiós a la verdad. Barcelona: Gedisa. 